\title{
Preservação da veia safena magna na cirurgia de varizes dos membros inferiores
}

\author{
Varicose vein surgery in lower limbs with preservation of the great \\ saphenous vein
}

Hamilton Almeida Rollo ${ }^{1}$, Mariângela Giannini ${ }^{2}$, Winston Bonetti Yoshida ${ }^{1}$

\begin{abstract}
Resumo
A veia safena magna autóloga é o melhor substituto arterial nas revascularizações dos membros inferiores, importante na revascularização do miocárdio e pode ser utilizada nas cirurgias do sistema venoso e nos traumas das extremidades. A fleboextração aumenta os riscos de lesões linfáticas e neurológicas. Assim, no tratamento das varizes primárias dos membros inferiores por meio da cirurgia ou de outras técnicas, a preservação da safena é recomendável se ela for normal ou apresentar alterações que ainda permitam sua preservação pela correção da causa desencadeante. Tal correção pode ser feita por técnicas cirúrgicas. Entre elas, a cura hemodinâmica da insuficiência venosa em ambulatório (CHIVA) tem mostrado bons resultados. Recentemente, um ensaio clínico randomizado e controlado foi publicado comprovando sua eficácia. Outra técnica bastante utilizada é a da ligadura rasante da junção safenofemoral + crossectomia + ligadura das tributárias de crossa, com a qual se tem obtido resultados contraditórios. Finalmente, as técnicas que corrigem a insuficiência da safena reparando as valvas ostial e pré-ostial (valvoplastia externa) são mais fisiológicas. Um ensaio clínico internacional multicêntrico, randomizado e controlado, testando um novo dispositivo, está sendo realizado, com resultados iniciais favoráveis. Este estudo pretende fazer uma revisão sobre as técnicas utilizadas na preservação da safena magna.
\end{abstract}

Palavras-chave: Varizes, veia safena magna, ultrassom, cirurgia.

\section{Introdução}

Nas 3 últimas décadas, o tratamento cirúrgico das varizes primárias dos membros inferiores foi motivo de grande atenção, tendo-se a preocupação de preservar a veia safena magna (VSM) sempre que possível, para eventual uso no futuro, como substituto arterial. A vida média das pessoas tem aumentado, com consequente aumento da população de idosos e maior chance de ocorrência das complicações de doenças degenerativas como a aterosclerose em nível coronariano e nas artérias das extremidades.

\begin{abstract}
The autologous great saphenous vein is the most effective bypass choice for lower limb revascularization, playing an important role in myocardial revascularization, and can be used in venous system surgeries and extremity traumas. Stripping increases the risk of lymphatic lesions and nerve damage. Therefore, when surgery or other techniques are used to treat primary varicose veins in the lower limbs, preservation of the saphenous vein is a desirable objective whenever the vein remains healthy or with alterations that still allow preservation by correcting the cause. Such corrections can be performed surgically. Among the methods currently used, the ambulatory conservative hemodynamic correction of venous insufficiency has yielded good results. A randomized controlled trial confirming the efficacy of this procedure has been recently published. Another largely used technique is flush ligation of the saphenofemoral junction plus saphenous veins stripping and ligation of its tributaries at the groin, which has yielded conflicting results. Finally, there are techniques that correct saphenofemoral reflux by external valvuloplasty of the saphenofemoral junction. An international multicenter, randomized, controlled trial, testing a new device, is currently in progress with favorable preliminary results. This study aimed to review the techniques employed to preserve the great saphenous vein in varicose vein surgery.
\end{abstract}

Keywords: Varicose veins, great saphenous vein, ultrasound, surgery.

A VSM é comprovadamente o melhor substituto arterial nas revascularizações infrainguinais nos membros inferiores $^{1}$ e nas revascularizações do miocárdio. Depois da artéria mamária, ainda é a melhor opção para o cirurgião cardíaco $^{2,3}$. Também pode ser usada como derivação nas obstruções do sistema venoso profundo (cirurgia tipo Pal$\mathrm{ma})^{4}$, como substituto arterial ou venoso nos traumas vasculares das extremidades com lesões das artérias e veias profundas em nível fêmoro-poplíteo e bráquio-axilar ${ }^{5}$, e, eventualmente, como acesso vascular para hemodiálise.

1. Livre-docente. Professor adjunto, Disciplina de Cirurgia Vascular, Departamento de Cirurgia e Ortopedia, Faculdade de Medicina, Universidade Estadual Paulista Júlio de Mesquita Filho (UNESP), Campus de Botucatu, Botucatu, SP.

2. Doutora. Professora assistente, Disciplina de Cirurgia Vascular, Departamento de Cirurgia e Ortopedia, Faculdade de Medicina, UNESP, Campus de Botucatu, Botucatu, SP.

Não foram declarados conflitos de interesse associados à publicação deste artigo.

Artigo submetido em 16.05.08, aceito em 06.03.09.

J Vasc Bras. 2009;8(2):154-165.

Copyright (C 2009 by Sociedade Brasileira de Angiologia e de Cirurgia Vascular 
Um outro aspecto a ser considerado é que a não-fleboextração da VSM pode reduzir as lesões dos vasos linfáticos e do nervo safeno que acompanham a VSM, resultando em menor risco de alterações nervosas e linfáticas no pós-operatório da cirurgia de varizes ${ }^{6}$. Pode haver também uma redução de hematomas quando não se faz a retirada da VSM.

Levando em consideração os fatos acima mencionados, é recomendável a preservação da VSM quando se indica a cirurgia para tratamento das varizes primárias dos membros inferiores. Na prática clínica nas cirurgias de varizes, procurava-se preservar a VSM sempre que essa se mostrava normal ao exame clínico. Com o desenvolvimento dos métodos diagnósticos não-invasivos, a avaliação pré-operatória permitiu identificar com mais clareza as alterações da VSM. Entre nós, Luccas et al. ${ }^{7}$, após observarem a VSM com o Doppler de ondas contínuas através de técnica por eles desenvolvida, indicaram a preservação sem nenhum procedimento sobre a VSM nos pacientes com safena normal ou pouco alterada, fazendo exclusivamente a retirada dos trajetos varicosos e ligadura das perfurantes. Obtiveram bons resultados $(87,69 \%)$ a curto e médio prazo ( 2 a 7 anos de seguimento $)^{8}$.

Nos últimos 30 anos, diversas técnicas vem sendo propostas visando preservar a $\mathrm{VSM}^{9-15}$, mesmo quando essa apresenta alguma alteração. Na maioria das vezes, as alterações varicosas que acometem a VSM são causadas pelo refluxo na junção safeno-femoral (JSF) por insuficiência da valva ostial, que se prolonga para o seu tronco e muitas vezes transfere-se para suas veias colaterais superficiais, as quais se tornam varicosas, mantendo a VSM em si pouco alterada ${ }^{16}$.

Desse modo, as técnicas para preservação da VSM tentam bloquear o refluxo na JSF ou corrigir a incompetência da valva ostial. Outras vezes, a valva da JSF é competente e as alterações da safena são provocadas por refluxo oriundo de outras fontes, como de perfurantes insuficientes que se conectam a ela, especialmente a perfurante de Hunter, situada no terço médio da coxa ou de transferência de refluxo através de veias colaterais que conectam a VSM à safena parva, por insuficiência da junção safeno-poplítea $(\text { JSP })^{17-19}$.

O refluxo para a VSM pode vir também de veias pélvicas ou da parede abdominal através das veias tributárias da crossa da safena (circunflexa ilíaca superficial, veia epi- gástrica superficial ou pudendas externas). Normalmente, o fluxo de tais veias direciona-se para a veia femoral comum, e isso ocorre devido à presença da valva subostial da $\mathrm{VSM}^{20}$. Quando a valva ostial está competente e a subostial não, pode haver inversão de fluxo, e se o refluxo é importante e não há uma perfurante de reentrada para $o$ sistema venoso profundo, há possibilidade de alterações na VSM. Stehling et al. ${ }^{21}$ encontraram essa alteração em $10 \%$ das VSM que tinham algum refluxo troncular, ou seja, o refluxo era ao nível da crossa, porém, por incompetência da valva subostial e não da ostial. Tais autores submeteram 41 pacientes com esse tipo de alteração à cirurgia, fazendo a ligadura e remoção das tributárias da crossa na maior extensão possível e tiveram bons resultados na maioria dos pacientes (92\%), em seguimento de curto prazo (2 a 18 meses).

Para que as alterações acima citadas sejam identificadas e se possa determinar qual a melhor técnica para a correção das mesmas (preservando-se a VSM), são importantes e necessários métodos adequados de diagnóstico que permitam a avaliação dos pacientes com varizes de membros inferiores, e que mostrem as anormalidades anatômicas e funcionais dos sistemas venosos, profundo (SVP) e superficial. Dentre os métodos auxiliares de diagnóstico o mapeamento dúplex em escala colorida (MDC)é atualmente o mais sensível, além da vantagem de ser não-invasivo. Ele permite caracterizar a normalidade ou não do SVP e os locais de refluxo das veias profundas para as superficiais (JSF, JSP e das veias perfurantes), bem como a distribuição anatômica das alterações varicosas no sistema superficial ${ }^{22-29}$. Possibilita, também, avaliar os diâmetros da VSM e se a mesma é retilínea ou tortuosa. Recentemente, alguns autores ${ }^{30,31}$ têm, inclusive, correlacionado as alterações de diâmetros da VSM pelo MDC, com volume de refluxo e gravidade do quadro clínico, parâmetros, esses, calculados pelo próprio MDC ou pela pletismografia a ar (PGA). Assim, parece possível se predizer qual o grau de alteração da VSM e se ela ainda pode ser preservada ou extirpada. Portanto, o MDC faz uma avaliação apropriada da fisiopatologia da doença varicosa, confirma se as varizes são de etiologia primária (SVP normal) e se o grau das alterações da VSM permite ainda que ela seja preservada.

As principais técnicas cirúrgicas para tratamento das varizes primárias com preservação da VSM que têm sido 
propostas e com resultados publicados na literatura são: ligadura da junção safeno-femoral (JSF), sem as ligaduras das veias tributárias da crossa ${ }^{11,32}$; ligadura e secção da JSF (crossectomia) + ligadura das tributárias da crossa ${ }^{10,12,33-39}$; valvoplastia das valvas ostial e sub-ostial da VSM, sem a ligadura da $\mathrm{JSF}^{13}$; e colocação de cuff (manguito) de politetrafluoretileno (PTFE) ou teflon envolvendo as valvas ostial e subostial, sem a ligadura da JSF ${ }^{14,15,40}$.

Pelo exposto acima, o presente artigo tem como objetivo fazer uma revisão sobre as principais técnicas que são utilizadas para preservação da safena magna.

\section{Revisão da literatura}

Das técnicas que preservam a VSM com ligadura da JSF e sem ligar as tributárias da crossa, a chamada CHIVA (cura hemodinâmica da insuficiência venosa em ambulatório) tem sido frequentemente realizada, principalmente na Europa (França, Itália e Espanha) (Tabela 1). Tal técnica foi idealizada por Franceschi ${ }^{11}$, em 1988, e basicamente consiste na ligadura da VSM com refluxo rente à veia femoral. Para manter a reentrada do sangue no SVP, evita-se fazer ligaduras das tributárias da crossa e das veias perfurantes insuficientes que se conectam à VSM. Para fracionar a coluna de pressão sanguínea, outras ligaduras no tronco da VSM com refluxo são feitas, de forma escalonada, imediatamente distais à conexão de perfurantes insuficientes. Faz-se também a interrupção dos shunts venovenosos que se ligam à VSM.

Em 1990 foi publicado por Mandolesi et al..$^{32}$ um estudo multicêntrico prospectivo e não-randomizado feito na Itália, onde 430 pacientes foram operados pela técnica CHIVA e seguidos em média por 14 meses. Foram obtidos bons resultados em $85 \%$ dos casos com ocorrência de tromboflebite de VSM no pós-operatório em $9 \%$ deles. No final da década de 90 , Cappelli et al. $^{41}$, num estudo nãorandomizado, e com seguimento de 3 anos, compararam os resultados da técnica CHIVA (148 pacientes), com os da safenectomia referidos por outros autores e com mesmo tempo de seguimento. Verificaram bons resultados, não diferindo das séries publicadas dos pacientes safenectomizados. Zamboni et al. ${ }^{42}$ estudaram prospectivamente de modo não-randomizado, 357 pacientes operados pela técnica CHIVA por período médio de 49 meses, sendo os mesmos avaliados no pré e pós-operatório com MD, fotopletismografia (FPG) e pela clínica. Em $94 \%$ dos casos, a

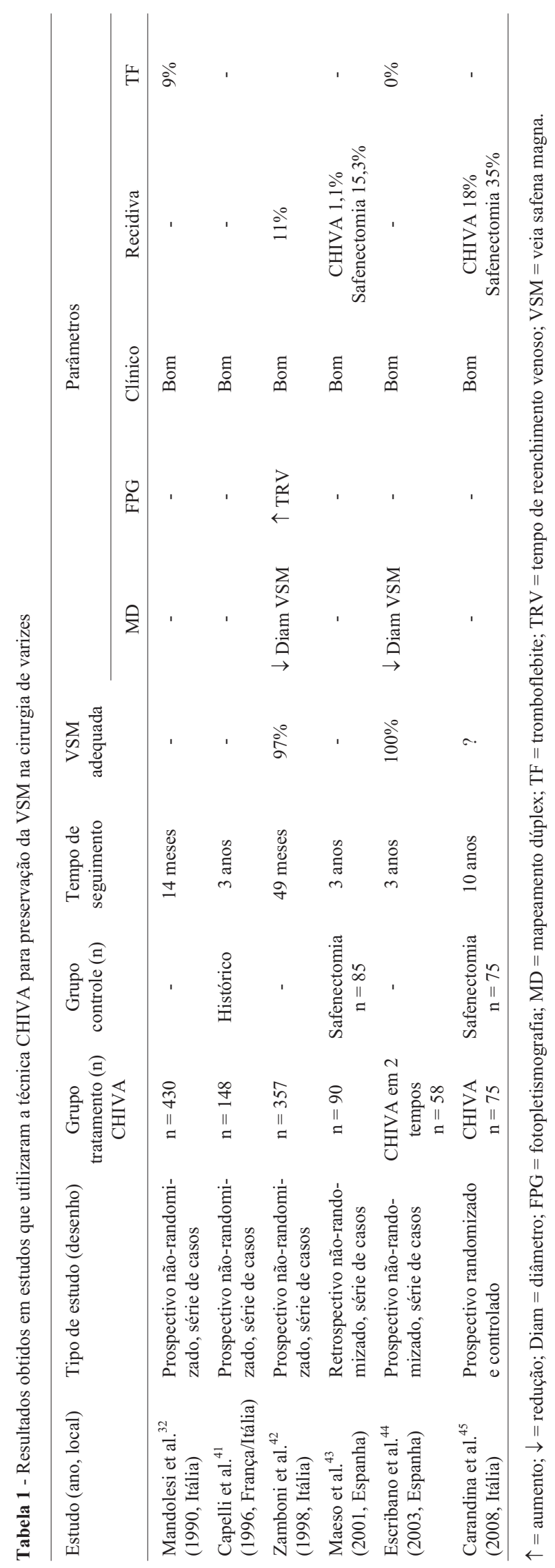


VSM estava preservada de modo adequado para ser utilizada como substituto arterial e com $11 \%$ de recidivas de veias varicosas.

Maeso et al. $^{43}$ publicaram um estudo retrospectivo não-randomizado, em que comparam pacientes operados pela técnica CHIVA com aqueles submetidos à safenectomia, com seguimento de 3 anos e avaliação clínica. Tiveram resultados melhores nos pacientes submetidos ao CHIVA, inclusive com menor ocorrência de recidivas. Mais recentemente, Escribano et al. ${ }^{44}$ utilizaram a técnica CHIVA em dois tempos: num primeiro tempo, ligando ou desconectando a origem da colateral insuficiente (shunt venovenoso), que continha a perfurante de reentrada na VSM. Se o refluxo na VSM não fosse resolvido, num segundo tempo se fazia a ligadura rasante de JSF. Obtiveram bons resultados clínicos em $89,6 \%$ dos casos e com redução do diâmetro de VSM na maioria dos pacientes.

Os estudos que utilizaram a técnica CHIVA e acima referidos são estudos de curto ou médio prazo, não-randomizados e não-controlados. Entretanto, Carandina et al. ${ }^{45}$ recentemente publicaram ensaio clínico randomizado e controlado comparando a técnica CHIVA com a cirurgia de varizes acompanhada de safenectomia. O estudo foi feito com 150 pacientes, ao longo de 10 anos, sendo 75 sorteados para CHIVA e 75 para safenectomia. Os pacientes submetidos a técnica CHIVA tiveram índice menor de recidivas do que os submetidos à safenectomia $(\mathrm{p}<0,04)$. A evolução clínica foi semelhante nos 2 grupos.

Em nosso serviço no Hospital das Clínicas da Faculdade de Medicina de Botucatu temos utilizado a técnica CHIVA com modificações ${ }^{46,47}$. As ligaduras escalonadas no tronco da VSM acima das perfurantes não são efetuadas e os trajetos varicosos retirados por mini incisões com a técnica de agulhas de crochê. Se houver conexão de trajetos varicosos à VSM, eles são ligados junto a mesma, assim preservando-se a VSM integralmente. As perfurantes insuficientes conectadas à VSM também são ligadas. Os resultados foram obtidos em estudo não-randomizado de uma série de casos, avaliados no pré-operatório pelo exame clínico e MDC, repetidos com 1, 3, 6 e 12 meses e anualmente a seguir no pós-operatório. Os resultados preliminares (até 2 anos) ${ }^{46}$ de 20 membros de 18 pacientes foram bons, com $77 \%$ dos pacientes com boa evolução clínica. A VSM foi preservada em toda sua extensão em 18 membros (90\%) e seus diâmetros médios diminuíram significante- mente $(\mathrm{p}<0,05)$ em relação ao pré-operatório, mas ainda adequadas para serem utilizadas como substituto arterial. Em dois membros houve oclusão da VSM ao nível da coxa por tromboflebite $(10 \%)$, porém, sendo ainda preservados $50 \%$ de extensão dessas veias. Na avaliação de médio prazo (4 a 6 anos) $)^{47}$ de 19 membros de 16 pacientes, 63\% deles ainda apresentavam boa evolução clínica e $89 \%$ apresentavam a VSM pérvia e adequada para enxerto em sua maior extensão. Recidiva de trajetos varicosos foi mais frequente em nível de perna ( $47 \%$ dos membros) e não relacionada à VSM, mas a perfurantes insuficientes.

A técnica cirúrgica que propõe a ligadura rasante da JSF, secção da crossa (crossectomia), ligadura de todas as tributárias da crossa e das perfurantes insuficientes, seguido de retirada de todos os trajetos varicosos por incisões escalonadas, tem sido proposta e utilizada por vários autores $^{10,12,33-39}$ (Tabela 2).

Em 1979, Jakobsen ${ }^{9}$ utilizou essa técnica modificada (associada à escleroterapia) em um ensaio clínico randomizado e controlado investigando 516 pacientes com varizes primárias, com insuficiência da VSM sem terem sofrido quaisquer tratamentos previamente. Os pacientes foram divididos em 3 grupos: no grupo 1 foi feita a safenectomia, ligadura das perfurantes e retirada dos trajetos varicosos por incisões escalonadas (cirurgia radical); no grupo 2, a ligadura da JSF, crossectomia e ligadura das tributárias + escleroterapia dos trajetos varicosos; e no grupo 3, apenas a escleroterapia. Os pacientes foram avaliados a curto e médio prazo ( 3 meses e 3 anos). Constatou-se que o grupo submetido à cirurgia radical apresentou melhor evolução clínica e menos recidivas (10,2\%), quando comparado ao grupo preservação $(34,8 \%)$.

O primeiro estudo, prospectivo, randomizado e duplo-cego publicado com essa técnica foi o de Munn et al. ${ }^{10}$. Os autores justificaram a realização do estudo, pois a técnica proposta por Rivlin ${ }^{52}$, estava sendo usada para tratamento das varizes na década de 70, e pretendia-se compará-la à técnica em que se fazia a safenectomia. A avaliação dos resultados foi clínica, feita por observadores nãopertencentes à equipe cirúrgica, e o seguimento foi de médio prazo (2,5 a 3,5 anos). Foram observados 57 pacientes com varizes bilaterais e simétricas, num total de 114 membros inferiores, e, desses, 57 foram sorteados para preservação da VSM e 57 foram para safenectomia. As recidivas de varizes ocorreram mais frequentemente nos membros 


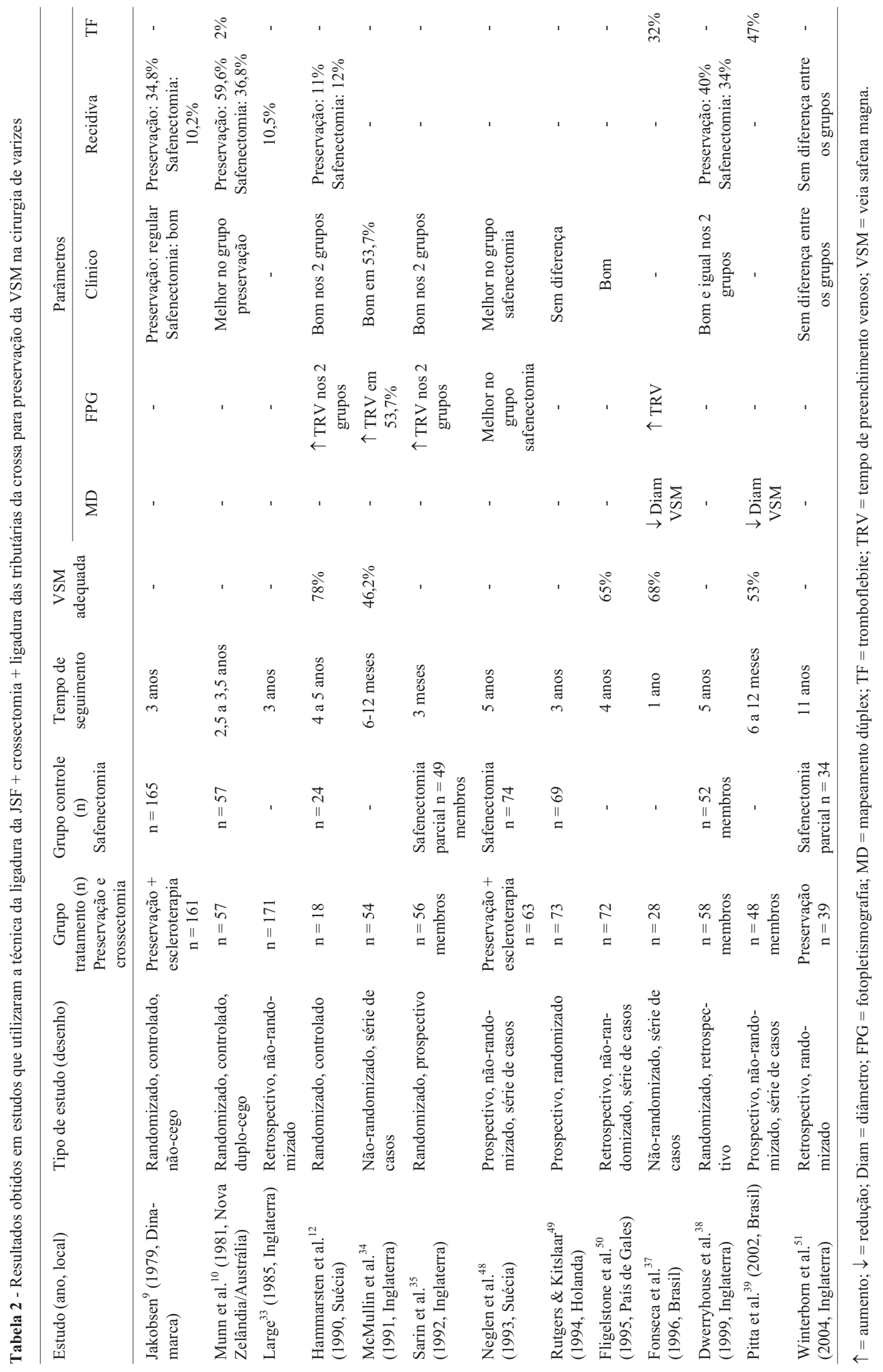


onde preservou-se a VSM $(59,6 \%)$ em relação aos que foram safenectomizados (36,8\%). Entretanto, a presença de parestesia foi maior nos pacientes submetidos à safenectomia, fazendo com que os pacientes operados com preservação da VSM mencionassem melhora clínica. Apesar do estudo ter sido randomizado e duplo-cego, a avaliação dos membros operados foi somente feita pela clínica, tanto no pré como no pós-operatório. Assim, não houve uma avaliação das VSM através de um método objetivo, como o MDC que pudesse determinar a gravidade e extensão das alterações das safenas, de modo a se ter um critério para se indicar a preservação da VSM. Tal falta de critério pode ter levado a maior incidência de recidivas nos membros em que foi preservada a safena, pois muitas VSM preservadas poderiam apresentar alterações graves, as quais não seriam indicativas de preservação. Por outro lado, Large ${ }^{33}$ obteve melhores resultados com essa técnica de preservação, tendo apenas 10,5\% de recidivas em seguimento de 3 anos. Seu estudo, porém, foi retrospectivo.

Hammarsten et al. ${ }^{12}$ realizaram um estudo randomizado e controlado, onde os pacientes, após serem avaliados por flebografia ascendente e descendente e pela pletismografia strain-gauge, foram operados e submetidos a ligadura + secção da JSF e das tributárias da crossa. Logo após, foram sorteados para safenectomia ou preservação da VSM. Em ambos os grupos, foram feitas retiradas dos trajetos varicosos e ligadura de todas as perfurantes insuficientes. Os pacientes foram reavaliados após 4 a 5 anos, através do exame clínico, pletismografia strain-gauge e pelo MDC, sendo verificados os diâmetros e perviedade da VSM. A maioria dos pacientes teve boa evolução clínica, a ocorrência de recidivas foi igual nos 2 grupos e a pletismografia voltou a valores normais. Não houve diferença estatística entre os dois grupos. No grupo em que a VSM foi preservada, $78 \%$ delas estavam adequadas para serem utilizadas como enxerto.

McMullin et al. ${ }^{34}$ em estudo prospectivo, não-randomizado e não-controlado de uma série de pacientes (54 membros inferiores), utilizou a fotopletismografia (FPG) e o MDC, antes e após (6 a 12 meses) a cirurgia de varizes, em que preservaram a VSM com a técnica de ligadura da $\mathrm{JSF}+$ secção da crossa e ligadura das tributárias. Após a cirurgia, o MD mostrou que em 2 dos 54 membros estudados, a JSF ainda estava aberta e com refluxo, e nos outros 52, a VSM não foi identificada em 28 (53,8\%) e estava pérvia em 24 membros (46,2\%). Nos 28 membros em que a VSM não foi visibilizada, os autores consideraram ter ocorrido trombose da veia ou retirada cirúrgica inadvertida. As 24 que foram identificadas ao MDC estavam pérvias e apresentavam fluxo retrógrado. Assim, essa técnica permitiu preservar apenas 50\% das VSM, índice não satisfatório para o objetivo de se ter a VSM em condições adequadas para uso no futuro como substituto arterial.

Sarin et al. ${ }^{35}$, com o objetivo de comparar a técnica utilizada por McMullin et al. ${ }^{34}$ para preservação da VSM, e a técnica que faz a safenectomia parcial ao nível da coxa até o terço superior da perna, fizeram um estudo prospectivo e randomizado em 105 membros inferiores de 69 pacientes com insuficiência da VSM por refluxo na JSF e que se estendia para o tronco da mesma. Verificaram a frequência de complicações neurológicas e a presença de refluxo residual na VSM preservada em nível da perna. Após o sorteio, 49 membros foram submetidos a safenectomia parcial e 56 a preservação total da VSM. Os pacientes foram avaliados clinicamente, pelo MDC e pela FPG antes e após 3 meses da cirurgia. A FPG mostrou melhora após a cirurgia e não houve diferença entre os dois grupos. O MDC revelou a presença de fluxo retrógrado nas VSM preservadas em nível da perna, sendo mais frequente nos membros com preservação total. Os autores não se referiram a aspectos relacionados à perviedade ou adequação para uso como substituto arterial. Não houve diferença entre os grupos quanto às complicações neurológicas; entretanto, o tempo de seguimento foi curto, impossibilitando a observação da ocorrência de recidivas. Assim, o interesse primordial deste trabalho está no estudo objetivo feito através do MDC e FPG antes e após a cirurgia.

Fligelstone et al. ${ }^{36}$, como os demais autores acima citados, estudaram a evolução a curto prazo de 44 pacientes num total de 75 membros, em que aplicaram a técnica descrita por Hammarsten et al. para preservação da VSM. Foi um estudo não-controlado de uma série de pacientes avaliados clinicamente por um observador independente e pelo MDC. No MDC, verificou-se a extensão, diâmetros e perviedade da VSM preservada, sendo os pacientes estudados de 6 a 14 meses, após a cirurgia. Encontraram VSM adequadas para serem usadas como substituto arterial em todo seu comprimento em $68 \%$ dos membros e na região da perna em $82 \%$. Não houve associação entre a presença de fluxo retrógrado na safena preservada e piora clínica. Pos- 
teriormente, Fligelstone et al..$^{50}$ avaliaram 72 membros desses pacientes mais tardiamente ( 3 a 5 anos) e verificaram que $65 \%$ das VSM estavam adequadas para serem utilizadas como enxerto. Além disso, a maioria dos pacientes estava tendo boa evolução clínica.

Neglen et al. ${ }^{48}$ compararam em estudo prospectivo e não-randomizado a técnica de Hammarsten et al. ${ }^{12}$ associada à escleroterapia dos trajetos varicosos, através de um grupo de pacientes submetidos a cirurgia radical (safenectomia) e outro tratado apenas pela escleroterapia. Os pacientes foram seguidos durante 5 anos, sendo observados clinicamente e pela FPG, e não pelo MDC. O grupo de pacientes submetidos a cirurgia radical teve melhores resultados tanto na avaliação clínica, como pela FPG.

Rutgers \& Kitslaar $^{49}$, de modo prospectivo e randômico, compararam pacientes portadores de varizes primárias com insuficiência da VSM submetidos a cirurgia radical, com pacientes com o mesmo diagnóstico e tratados pela técnica de Hammarsten et al. associada a escleroterapia compressiva dos trajetos varicosos. Os pacientes foram avaliados no PO com 3 meses, e com 1, 2 e 3 anos, pela clínica + Dopplerometria de ondas contínuas. Se durante as verificações fossem notados trajetos varicosos residuais ou recidivados, eles eram tratados pela escleroterapia. No final dos 3 anos, o grupo tratado pela cirurgia radical teve resultados cosméticos melhores, porém não houve diferença entre os grupos quanto à satisfação dos pacientes em relação ao tratamento.

Dwerryhouse et al. ${ }^{38}$ realizaram estudo randomizado comparando a técnica da ligadura rasante e secção da JSF + ligadura das tributárias da VSM associada a safenectomia ou não (Hammarsten et al. ${ }^{12}$ ), sendo feito o sorteio após a ligadura da JSF. Foram operados 78 pacientes num total de 110 membros, dos quais 52 foram submetidos a safenectomia da VSM até o joelho, e em 58 a safena foi preservada. Os pacientes foram revistos 5 anos após a cirurgia através do exame clínico e MDC. A maioria dos pacientes teve boa evolução clínica (83\%) e não houve diferença estatística entre os grupos. Também não houve diferença estatística entre os grupos em relação à recorrência de varizes ( $34 \%$ nos safenectomizados e $40 \%$ nos que a VSM foi preservada). Entretanto, o número de reoperações foi maior nos pacientes da preservação, por recorrência de insuficiência da JSF. Recentemente, em 2004, 51 pacientes deste estudo foram reavaliados a longo prazo (11 anos).
Verificou-se que não havia diferença entre os grupos quanto a recidiva de varizes ${ }^{51}$.

Entre nós, Fonseca et al. ${ }^{37}$ e mais recentemente Pitta et al. $(2002)^{39}$ utilizaram a técnica descrita por Hammarsten et al. ${ }^{12}$, sendo que Pitta et al. ${ }^{39}$ modificaram a técnica, pois, além de ligarem as tributárias da crossa, também as extirparam. Como Hammarsten et al. ${ }^{12}$, tais autores demonstraram resultados favoráveis a curto prazo, porém observaram na avaliação precoce (de 1 a 3 meses), ocorrência de trombose na VSM no segmento da coxa em 30 a $50 \%$ dos casos. Apesar de referirem boa recanalização desses segmentos posteriomente ( 6 a 12 meses), há dúvida quanto à adequação dessas veias para enxerto no futuro.

Belcaro et al. ${ }^{53}$ fizeram um estudo prospectivo e randomizado de pacientes com varizes dos membros inferiores que apresentavam insuficiência da VSM por refluxo na JSF, empregando duas técnicas diferentes de preservação da VSM. Num dos grupos (369 pacientes), foi realizada a técnica de ligadura rasante e secção da JSF + ligadura das tributárias da crossa e no outro (377 pacientes) foi feita apenas a ligadura e secção da VSM 3 a 5 cm abaixo da JSF, sem serem ligadas as tributárias da crossa. Os pacientes foram vistos no pré e pós-operatório (10 anos após a cirurgia), através da medida da pressão venosa ambulatória (PVA), tempo de enchimento venoso (TEV) e pelo MD. Também no pós-operatório foram feito exames clínicos e pelo MDC, anualmente. Caso os pacientes tivessem varizes residuais ou recidivadas, elas eram tratadas por escleroterapia. No final de 10 anos, os grupos mostraram resultados semelhantes, sugerindo que a ligadura + secção da VSM distal à JSF é uma boa alternativa para se preservar a VSM, apesar de, teoricamente, esse procedimento não parecer adequado por não corrigir o refluxo na JSF, o que poderia propiciar dilatação das tributárias da crossa.

Finalmente, existem técnicas que preservam a VSM sem haver necessidade de ligadura da JSF; porém, corrigindo a insuficiência das valvas da JSF por alguma técnica de valvoplastia que permita a normalização do retorno venoso pela VSM sem bloqueio ao nível da junção. Essas técnicas parecem uma alternativa mais fisiológica para correção da insuficiência das valvas da JSF. É importante que se considere o fato de saber se as valvas da JSF ainda podem ser reparadas. Isso pode ser conseguido por meio do ultrassom de imagem. Duas técnicas têm sido propostas para correção da disfunção valvar: numa delas, se faz a 
valvoplastia externa da valva ostial na JSF através de sutura longitudinal ou plicatura para reduzir a luz da veia na altura da valva para $60-70 \%$, permitido assim, que as cúspides se fechem por completo restaurando a competência da mesma. Na outra, a valvoplastia externa da JSF é feita pela colocação de cuff (manguito) de PTFE ou teflon nos locais das valvas, afim de diminuir o lúmem e torná-las novamente competentes. Na Tabela 3 são mostrados os principais trabalhos que utilizaram essas técnicas, e, em seguida, eles são analisados.

Belcaro, em $1993^{13}$, publicou um estudo prospectivo e randomizado, no qual 54 pacientes com insuficiência da VSM por refluxo na JSF que se estendia para o tronco da mesma foram divididos por sorteio em dois grupos. No primeiro aplicou a técnica da plicatura para correção da valva da JSF e no outro (grupo controle) nenhum tratamento. Os pacientes foram examinados no pré e pós-operatório pelo MDC e pela medida da PVA + TRV. Após 5 anos, 43 pacientes, (22 no grupo tratamento e 21 no controle) foram avaliados. No grupo controle todos pioraram, enquanto que no grupo sob tratamento, $68,25 \%$ que não tinham refluxo na JSF melhoram os parâmetros obtidos e a maioria estava tendo boa evolução clínica. Nesse grupo, em 31,8\% dos pacientes, nos quais o refluxo não foi corrigido, os parâmetros não melhoraram e todos estavam tendo evolução clínica não-satisfatória.

A respeito da técnica da valvuloplastia com cuff, Schanzer \& Skladany ${ }^{40}$ compararam duas séries de pacientes de modo não-randomizado, com insuficiência da VSM por refluxo na JSF e troncular, sendo que na primeira série (12 pacientes, 15 membros) foi feita a valvoplastia externa da JSF com pequeno manguito de dácron reforçado com silicone. Na segunda série, 14 pacientes (16 membros) foram submetidos à ligadura rasante da JSF. Em ambas as séries as tributárias da crossa da VSM foram ligadas e seccionadas, todos os trajetos varicosos retirados por incisões escalonadas e as VSM preservadas. Os pacientes foram examinados antes da cirurgia e após 1 e 6 meses através do MDC e também do ponto de vista clínico. Posteriormente, foram seguidos até 1 ano apenas pela clínica. Neste período não foram observadas recidivas e a evolução clínica foi boa em ambos os grupos. O MDC mostrou perviedade total da veia safena em $87 \%$ dos membros do grupo tratado com valvoplastia externa e de apenas $12,5 \%$ no grupo com ligadura da JSF. Assim, a tromboflebite ocorreu em 13\%

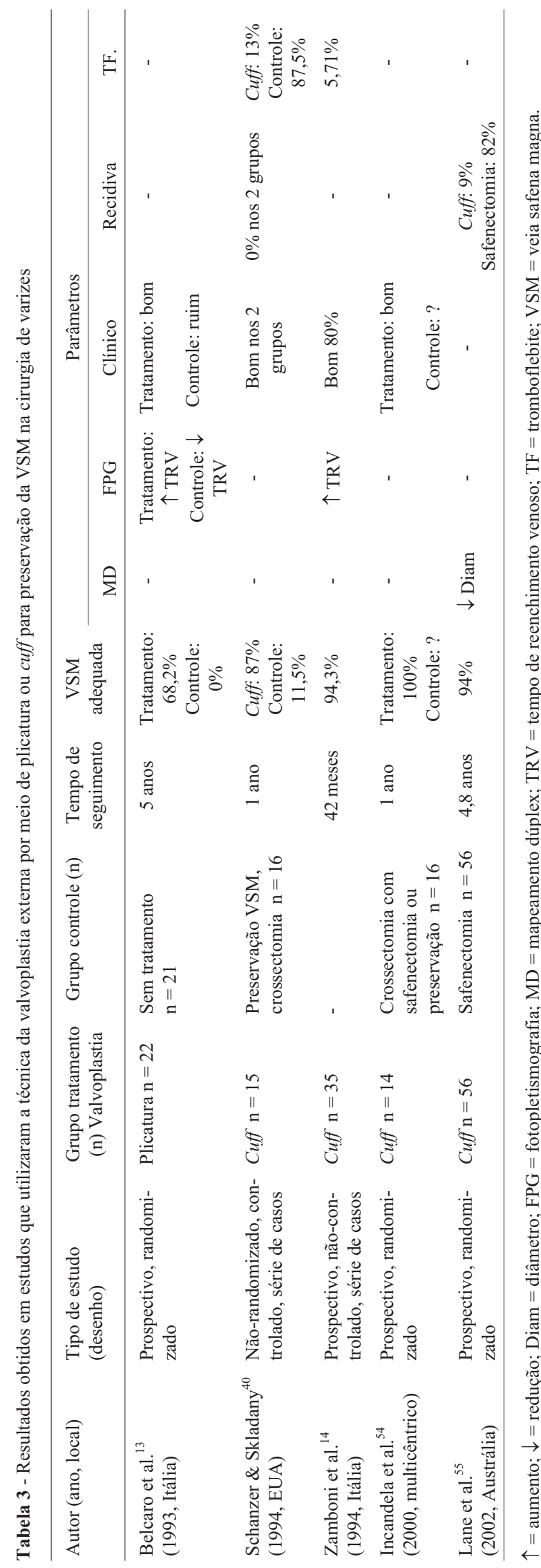


no grupo da valvoplastia e $87,5 \%$ no grupo da ligadura. Dos 13 pacientes submetidos à valvoplastia, sem ocorrência da tromboflebite, em 1 caso a valvoplastia falhou e não corrigiu o refluxo.

Zamboni et al. ${ }^{14}$ estudaram uma série de 35 pacientes portadores de varizes primárias que foram selecionados por apresentarem refluxo na JSF e valvas ostial e pré-ostial com as cúspides ainda preservadas, quando verificadas pelo MDC. Foi um estudo não-controlado, em que os pacientes foram seguidos por até 42 meses sendo feita avaliação no pré e pós-operatório através do MDC. Em 15 pacientes também foi realizada PVA e TRV por meio da fotopletismografia antes e após a cirurgia (uma semana). $\mathrm{Na}$ maioria dos pacientes, o refluxo na JSF foi corrigido por valvoplastia externa com manguito de PTFE sobre as valvas ostial e pré-ostial. Os pacientes tiveram boa evolução clínica, a VSM ficou totalmente pérvia em 33 (94,3\%); houve tromboflebite em duas $(5,71 \%)$ e em outras duas houve recorrência do refluxo na JSF. Nos 15 pacientes que foram examinados pela medida da PVA e TRV, os valores alterados no pré- operatório voltaram ao normal após a cirurgia havendo diferença estatística entre eles. Em 1998, Zamboni et al. ${ }^{42}$ publicaram os resultados de um estudo prospectivo de uma série de pacientes nos quais foram realizadas 64 valvuloplastias externas na JSF que apresentavam válvulas insuficientes, porém, com cúspides ainda preservadas. Dessas, 42 foram realizadas pela técnica da sutura externa ou plicatura e 22 com cuff de PTFE e o seguimento médio dos pacientes foi de 52 meses. Foram observadas $94 \%$ de safenas totalmente pérvias e recorrência de varizes de $12 \%$.

Incandela et al. ${ }^{54}$ publicaram os resultados iniciais e parciais de um estudo multicêntrico internacional prospectivo e randomizado que tem por objetivo avaliar um novo dispositivo (Gore External Vein Support-EVS) para se realizar a valvuloplastia da JSF. O estudo se desenvolve em 10 centros europeus (20 pacientes por centro) e o principal critério de inclusão é insuficiência da JSF e da VSM + presença de valva da JSF com cúspides de aspecto normal e funcionantes. Os pacientes com varizes primárias de grau leve ou moderado são sorteados para o grupo EVS ou controle (ligadura rasante da JSF, das tributárias e com ou sem safenectomia). As veias varicosas são retiradas por incisões escalonadas e perfurantes insuficientes ligadas. Os pacientes são avaliados clinicamente, pelo MDC e pela
PVA ou pletismografia a ar no pré e pós-operatório com 1 e 6 meses, e anualmente até 3 anos. Os autores publicaram os resultados dos primeiros 30 pacientes, sendo 14 do grupo EVS e 16 controles. No grupo EVS todas as safenas estavam pérvias, não havia refluxo na JSF, com boa evolução clínica e sem complicações (sem tromboflebite ou infecção do dispositivo). Assim, os resultados iniciais com o EVS estão sendo eficazes e seguros.

Lane et al. desenvolveram um stent valvar externo (Venocuff) $)^{15}$ para correção da insuficiência das valvas ostial e subostial da JSF, que posteriomente foi aperfeiçoado $(\text { Venocuff II })^{55}$. Apresentaram os resultados a curto e médio prazo da aplicação desse dispositivo em 2002: Verificaram que na avaliação, 3 meses após o implante, $94 \%$ das JSFs estavam competentes e após 4,8 anos $90 \%$ continuavam competentes. Os pacientes foram estudados no pré e pós-operatório pelo MDC, fotoplestimografia e também do ponto de vista clínico, sendo o estudo prospectivo, porém, não-randomizado. Os diâmetros da VSM reduziram significativamente e os pacientes tiveram boa evolução clínica e ocorrência de recidiva baixa. Mais recentemente, estes autores publicaram resultados a longo prazo dessa técnica, que se mantiveram bons ${ }^{56}$.

\section{Considerações finais}

Quando é feito o tratamento das varizes primárias dos membros inferiores por meio da cirurgia ou de outros procedimentos, como a esclerose compressiva, a preservação da VSM é recomendável sempre que possível, ou seja, se ela estiver normal ou com alterações que ainda permitam a sua recuperação pela correção ou eliminação da causa que está desencadeando a sua disfunção. Essa correção tem sido realizada através da aplicação de técnicas cirúrgicas, entre as quais, a denominada CHIVA $^{11}$ utilizada por alguns cirurgiões ${ }^{32,41-44,47}$. Com ela, tem-se obtido bons resultados a curto e médio prazo e recentemente estudo randomizado e controlado comprovou a eficácia dessa técnica $^{45}$.

Das técnicas que preservam a VSM, corrigindo o refluxo na JSF por ligadura rasante da mesma + crossectomia e ligadura das tributárias da crossa, os estudos (muitos deles randomizados e controlados, e outros com avaliação por métodos diagnósticos objetivos) têm mostrado resultados contraditórios ${ }^{10,12,33-39}$. Nesses estudos, a preservação da VSM nem sempre é obtida em uma extensão adequada 
para enxerto ou o índice de recidiva, a médio e longo prazo, não é satisfatório. Mais recentemente, em relação à recidiva, tem sido proposto ${ }^{57,58}$ que os pacientes sejam seguidos mais de perto, incluindo procedimentos coadjuvantes para controle dos trajetos varicosos recidivados, como cirurgia ambulatorial para retirada dos trajetos por mini-incisões escalonadas ou escleroterapia compressiva, mas mantendo-se a VSM preservada.

As técnicas que corrigem a insuficiência da VSM reparando as válvulas da JSF (valvuloplastia externa) parecem mais fisiológicas por não bloquearem a drenagem venosa através da JSF, que segundo alguns autores ${ }^{55}$ seria estímulo para surgimento de veias colaterais avalvuladas as quais propiciariam recidivas das varizes. Um ensaio clínico multicêntrico, randomizado e controlado, testando dispositivo para valvuloplastias da JSF está em andamento na Europa ${ }^{54}$ e os resultados iniciais publicados parecem ser favoráveis.

Do mesmo modo que para o tratamento das varizes dos membros inferiores, para o qual não existe nível de evidência, as técnicas para correção da insuficiência da VSM, com preservação da mesma, também necessitam de mais evidências de sua eficácia. Entretanto, devem ser consideradas questões éticas, ou seja, se a preservação da VSM pode ser importante para uma futura necessidade como enxerto ou substituto arterial, como submeter o paciente a um sorteio que pode resultar na perda da VSM ainda possível de ser preservada?

\section{Referências}

1. Veith FJ, Gupta SK, Ascer E, et al. Six year prospective multicenter randomized comparison of autologous saphenous vein and expanded polytetrafluorethylene grafts in infraiguinal arterial reconstructions. J Vasc Surg. 1986;3:104-14.

2. Goldman S, Zadina K, Moritz T, et al. Long-term patency of saphenous vein and left internal mammary artery grafts after coronary artery bypass surgery. Results from a Department of Veterans Affairs Cooperative Study. J Am Coll Cardiol. 2004;44:2149-56.

3. Tsui JC, Souza DS, Filbey D, Bomfim V, Dashwood MR. Preserved endothelial integrity and nitric oxide synthase in saphenous vein grafts harvested by a "no-touch" technique. Br J Surg. 2001;88:1209-15.

4. Al-Assal F, Gomes ACS, Al-Assal R. Tratamento cirúrgico da hipertensão venosa crônica. In: Maffei FHA, Lastória S, Yoshida WB, Rollo HA. Doenças vasculares periféricas. $3^{\mathrm{a}}$ ed. São Paulo: Medsi; 2002.
5. Burihan E, Poli de Figueiredo LF. Traumatismos vasculares. In: Maffei FHA, Lastória S, Yoshida WB, Rollo HA. Doenças vasculares periféricas. $3^{\mathrm{a}}$ ed. São Paulo: Medsi; 2002.

6. Holme JB, Skajaa K, Holme K. Incidence of lesions of the saphenous nerve after partial or complete stripping of the long saphenous vein. Acta Chir Scand. 1990;156:145-8.

7. Luccas GC, Kauffman P, Lane JC. Método de avaliação do estado funcional da veia safena interna. Cir Vasc Angiol. 1985;1:22-8.

8. Luccas GC, Parente JBF, Nagase Y, Lane JC. Preservação da veia safena magna em cirurgia de varizes: resultados tardios. Cir Vasc Angiol. 1995;11:15-8.

9. Jakobsen BH. The value of different forms of treatment for varicose veins. Br J Surg. 1979;66:182-4.

10. Munn SR, Morton JB, Macbeth WAAG, Mcleish AR. To strip or not to strip the long saphenous vein? A varicose veins trial. Br J Surg. 1981;68:426-8.

11. Franceschi C. Pour une cure conservatrice et hemodynamique de l'insufficience veineuse en ambulatoire: CHIVA. Angiologie. 1988;72:99-100.

12. Hammarsten J, Pedersen P, Claes-Goran C, Campanello M. Long saphenous vein saving surgery for varicose veins. A long-term follow-up. Eur J Vasc Surg. 1990;4:361-4.

13. Belcaro G. Correction of superficial incompetence with selective saphenous vein repair (SSVR). A five-year follow-up study. Vasc Surg. 1993;27:122-7.

14. Zamboni P, Gasbarro V, Marcelino MG, et al. External valvuloplasty of the saphenofemoral junction. Vasc Surg 1994;28:327-36.

15. Lane RJ, McMahon C, Cuzzilla ML. The treatment of varicose veins using the venous valve cuff. Phlebology. 1994;9:136-45.

16. Zamboni P, Cappelli M, Marcelino MG, Murgia AP, Pisano L, Fabi P. Does a varicose saphenous vein exist? Phlebology. 1997;12:74-7.

17. Rollo HA. Mapeamento dúplex no diagnóstico das doenças venosas. In: Maffei FHA, Lastória S, Yoshida WB, Rollo HA. Doenças vasculares periféricas. $3^{\text {a }}$ ed. São Paulo: Medsi; 2002.

18. Myers KA, Wood SR, Lee V, Koh P. Variations of connections to the saphenous systems in limbs with primary varicose veins: a study of 1481 limbs by duplex ultrasound scanning. J Phlebol. 2002;2:11-7.

19. Sarquis AL. Avaliação pré e pós-operatória no tratamento cirúrgico conservador de varizes tronculares com o duplex scan a cores. Cir Vasc Angiol. 1996;12 Supl 4:9-11.

20. Cappelli M, Molino Lova R, Ermini S, Zamboni P. Hemodynamics of saphenous-femoral junction. Patterns of reflux and their clinical implications. Int Angiol. 2004;23:25-8.

21. Stehling AP, Sarquis AL, Miguel EV, Morais GM, Miranda PRT. Correção hemodinâmica do refluxo na croça da safena magna por insuficiência da válvula pré-ostial: análise prospectiva de 41 pacientes. Cir Vasc Angiol. 2000;16:197-203.

22. Hanrahan LM, Kechejian GJ, Cordts PR, et al. Patterns of venous insufficiency in patients with varicose veins. Arch Surg. 1991;126:687-91.

23. van der Heijden FH, Bruyninckx CM. Preoperative colourcoded duplex scanning in varicose veins of the lower extremity. Eur J Surg. 1993;159:329-33. 
24. Franco G. Echo-Doppler couleur et exploration veineuse superficielle. Phlébologie. 1994;47:63-75.

25. Luccas GC, Nagase Y, Meneses FH, et al. Cirurgia de varizes dos membros inferiores. Avaliação pré-operatória do sistema venoso com mapeamento duplex. Cir Vasc Angiol. 1996;12:15-20.

26. Labropoulos N, Belcaro G, Giannoukas AD, et al. Can the main trunk of greater saphenous vein be spared in patients with varicose veins? Vasc Surg. 1997;31:531-4.

27. Moraes Filho D, El Hozni Jr RA, Diniz JAM, et al. Uso do duplex ultrassom no planejamento do tratamento cirúrgico de varizes dos membros inferiores. Cir Vasc Angiol. 1999;16:43-9.

28. Cooper DG, Hillman-Cooper CS, Baker SGE, Hollingsworth SJ. Primary varicose veins: The sapheno-femoral junction, distribution of varicosities and patterns of incompetence. Eur J Vasc Endovasc Surg. 2003;25:53-9.

29. Wong JKF, Duncan JL, Nichols DM. Whole-leg duplex mapping for varicose veins: observations on patterns of reflux in recurrent and primary legs, with clinical correlation. Eur J Vasc Endovasc Surg. 2003;25:267-75.

30. Barel EV. Estudo quantitativo do refluxo da veia safena interna pelo dúplex em pacientes com varizes dos membros inferiores [tese de doutorado]. Campinas: Universidade Estadual de Campinas (UNICAMP); 2002.

31. Navarro TP, Delis KT, Ribeiro AP. Clinical and hemodynamic significance of the greater saphenous vein diameter in chronic venous insufficiency. Arch Surg. 2002;137:1233-7.

32. Mandolesi S, Ballo M, Galeandro I, et al. [The 1st national multicenter study of the CHIVA (Conservative Therapy and Hemodynamics in Venous Insufficiency in Outpatient Departments) method of treatment of varices. One-year follow-up.] Ann Ital Chir. 1990;61:425-7.

33. Large J. Surgical treatment of saphenous varices, with preservation of the main great saphenous trunk. J Vasc Surg. 1985;2:886-91.

34. McMullin GM, Coleridge Smith PD, Scurr JH. Objective assessment of high ligation without stripping the long saphenous vein. Br J Surg. 1991;78:1139-42.

35. Sarin S, Scurr JH, Coleridge Smith PD. Assessment of stripping the long saphenous vein in the treatment of primary varicose veins. Br J Surg. 1992;79:889-93.

36. Fligelstone L, Carolan G, Pugh N, Minst P, Shandall A, Lane I. An assessment of the long saphenous vein for potential use as vascular conduit after varicose vein surgery. J Vasc Surg. 1993; 18:836-40.

37. Fonseca FP, Evangelista SSM, Sarquis AL. O tratamento cirúrgico ambulatorial e com anestesia local das varizes tronculares primárias dos membros inferiores, com preservação das safenas: avaliação pré e pós-operatória com o duplex scan a cores e com a fotopletismografia. Cir Vasc Angiol. 1996;12:19-22.

38. Dwerryhouse S, Davies B, Harradine K, Earnshaw JJ. Stripping the long saphenous vein reduces the rate of reoperation for recurrent varicose veins: five-year results of a randomized trial. J Vasc Surg. 1999;29:589-92.
39. Pitta GB, Castro AA, Teixeira LR, Francisco Jr J, Miranda Jr. F, Burihan E. Preservação da veia safena magna na cirurgia de varizes tronculares primárias. J Vasc Bras. 2002;1:32-8.

40. Schanzer H, Skladany M. Varicose vein surgery with preservation of the saphenous vein: A comparison between high ligation-avulsion versus saphenofemoral banding valvuloplasty-avulsion. J Vasc Surg. 1994;20:684-7.

41. Cappelli M, Molino Lova R, Ermini S, Turchi A, Bono G, Franceschi C. [Comparison between the CHIVA cure and stripping in the treatment of varicose veins of the legs: follow-up of 3 years.] J Mal Vasc. 1996;21:40-6.

42. Zamboni P, Marcellino MG, Cappelli M, et al. Saphenous vein sparing surgery: principles, techniques and results. J Cardiovasc Surg (Torino). 1998;39:151-62.

43. Maeso J, Juan J, Escribano JM, et al. Comparison of clinical outcome of stripping and CHIVA for treatment of varicose veins in the lower extremities. Ann Vasc Surg. 2001;15:661-5.

44. Escribano JM, Juan J, Bofill R, Maeso J, Rodrígues-Mori A, Matas M. Durability of the reflux-elimination by a minimal invasive CHIVA procedure on patients with varicose veins. A 3 years prospective case study. Eur J Vasc Endovasc Surg. 2003;25:159-63.

45. Carandina S, Mari C, De Palma M, et al. Varicose vein stripping vs haemodynamic correction (CHIVA): a long term randomised trial. Eur J Vasc Endovasc Surg. 2008;35:230-7.

46. Rollo HA, Lastória S, Yoshida WB, Moura R, Maffei FA. Cirurgia de varizes com preservação da veia safena magna avaliação pré e pós-operatória pelo mapeamento duplex resultados preliminares. Cir Vasc Angiol. 1996:4:63-8.

47. Lastória S, Rollo HA. Tratamento das varizes de membros inferiores. In: Maffei FHA, Lastória S, Yoshida WB, Rollo HA. Doenças vasculares periféricas. $3^{\mathrm{a}}$ ed. São Paulo: Medsi; 2002.

48. Neglén P, Einarsson E, Eklof B. The functional long-term value of different types of treatment for saphenous vein incompetence. J Cardiovasc Surg (Torino). 1993;34:295-301.

49. Rutgers PH, Kitslaar PJ. Randomized trial of stripping versus high ligation combined with sclerotherapy in the treatment of the incompetent greater saphenous vein. Am J Surg. 1994;168:311-5.

50. Fligelstone LJ, Salaman RA, Oshodi TO, et al. Flush saphenousfemoral ligation and multiple stab phlebectomy preserve a useful greater saphenous vein four years after surgery. J Vasc Surg. 1995;22:588-92.

51. Winterborn RJ, Foy C, Earnshaw JJ. Causes of varicose vein recurrence: late results of a randomized controlled trial of stripping the long saphenous vein. J Vasc Surg. 2004;40:634-9.

52. Rivlin S. The surgical cure of primary varicose veins. Br J Surg. 1975;62:913-7.

53. Belcaro G, Nicolaides AN, Cesarone MR, et al. Flush ligation of the saphenofemoral junction vs simple, distal ligation, 10-year, follow-up. The safe study. Angeiologie. 2002;54:19-23.

54. Incandela L, Belcaro G, Nicolaides AN, et al. Superficial vein valve repair with a new external valve support (EVS): the IMES (International Multicenter EVS Study). Angiology. 2000;51:S39-52. 
55. Lane RJ, Cuzzilla ML, Coroneos JC. The treatment of varicose veins with external stenting to the saphenofemoral junction. Vasc Endovascular Surg. 2002;36:179-92.

56. Lane RJ, Graiche JA, Coroneos JC, Cuzzilla ML. Long-term comparison of external valvular stenting and stripping of varicose veins. ANZ J Surg. 2003;73:605-9.

57. Brethauer SA, Murray JD, Hatter DG, Reeves TR, Hemp JR, Bergan JJ. Treatment of varicose veins: proximal saphenofemoral ligation comparing adjunctive varicose phlebectomy with sclerothrapy at a military medical center. Vasc Surg. 2001;35:51-8.

58. Recek C. Saphenofemoral junction ligation supplemented by postoperative sclerotherapy: A review of long-term clinical and hemodynamic results. Vasc Endovascular Surg. 2004;38:533-40.

Correspondência:

Hamilton Almeida Rollo

Departamento de Cirurgia e Ortopedia da Faculdade de Medicina de Botucatu

Campus de Botucatu (UNESP)

Rubião Junior $\mathrm{s} / \mathrm{n}^{\mathrm{o}}$

CEP 18618-970 - Botucatu, SP

Tel.: (14) 3811.6269

E-mail: hrollo@fmb.unesp.br 